\title{
Erfolgsbedingungen ideologiekritischer Praxis und das Problem des Paternalismus
}

\author{
Patrick Körner
}

Keywords: Ideologiekritik, Aufklärung, Marxismus, Methodologie, Aufklärungstechnologie, Psychologie, Backfire Effects, Mündigkeit, Paternalismus

\begin{abstract}
Der praktische Anspruch von Ideologiekritik - die Reduzierung und Überwindung von Ideologien - kann nur durch die Berücksichtigung der Ergebnisse der empirischen Einzelwissenschaften und die Förderung der kritischen Kompetenzen der aufzuklärenden Akteure eingelöst werden. Am Beispiel der „Backfire Effects“ wird nachgewiesen, weshalb Aufklärungstechnologien und die Einschätzungen ihres Erfolgspotentials auf die empirischen und idealiter experimentell verfahrenden Einzelwissenschaften angewiesen sind. Das bei der Anwendung dieser Ergebnisse deutlich werdende Paternalismusproblem kann entschärft werden - und die Lösung dieses Problems verdeutlicht schließlich sowohl die zweckmäßige Auswahl des aufklärungstechnologisch einzusetzenden Sachwissens als auch die Grenzen eines allein auf klassische Aufklärungsarbeit setzenden Verständnisses praktischer Ideologiekritik.
\end{abstract}

Körner, Patrick. 2019. „Erfolgsbedingungen ideologiekritischer Praxis und das Problem des Paternalismus“. In Ideologie, Kritik, Öffentlichkeit. Verhandlungen des Netzwerks Kritische Kommunikationswissenschaft, herausgegeben von Uwe Krüger und Sebastian Sevignani, 348-372. Universität Leipzig. DOI: 10.36730/ideologiekritik.2019.16

Patrick Körner | Heinrich-Heine-Universität Düsseldorf | patrick.koerner@hhu.de 


\section{$1 \quad$ Einleitung}

Es ist eine Sache, Ideologien zu erkennen, ihren problematischen Charakter näher zu bestimmen und ihre systematische Entstehung und Persistenz zu erklären. Eine andere ist es, Methoden zu finden, die eine erfolgreiche Reduzierung bestehender und Verhinderung potentieller Ideologien ermöglichen. Eine solche Überwindung oder zumindest Reduzierung von Ideologien ist typischerweise der praktische Anspruch der Ideologiekritik. Der Begriff „Aufklärungstechnologie“ bezeichnet hier die Kontexte, in denen dieser praktische Anspruch eingelöst werden soll.

Das Ziel des vorliegenden Beitrags besteht darin, aufzuzeigen, dass eine solche Aufklärungstechnologie mit den empirischen Einzelwissenschaften kooperieren muss, wenn sie ihren Anspruch einlösen will. Zudem wird dafür argumentiert, dass eine bestimmte Form der klassischen Wissensvermittlung, wie sie in der Aufklärungsphilosophie als Hauptinstrument ideologiekritischer Aufklärungstechnologie angesehen wurde, für jede Aufklärungstechnologie zu berücksichtigen ist, die beansprucht, nicht nur Ideologien zu bekämpfen, sondern zugleich die Mündigkeit und damit die kritischen Kompetenzen der aufzuklärenden Akteure zu erhöhen. Aus dem Ziel der Förderung der Mündigkeit der Adressaten resultiert ein Paternalismusdilemma, das am Ende des Beitrags aufgelöst wird.

Anschließend an die Charakterisierung des Gegenstands der Aufklärungstechnologie wird ein Beispiel aus neueren Diskussionen in der angelsächsischen Psychologie herangezogen, das nahe legt, dass die Praxis der Irrtumskorrektur mit Problemen zu kämpfen hat, die auch ideologiekritische Aufklärungstechnologien dazu zwingt, neue Wege zu gehen. Im Fokus stehen hier sogenannte „Backfire Effects“. ${ }^{1}$

Die erste Hauptthese dieses Beitrags ist, dass Ideologiekritiken ihren praktischen Anspruch nur dann einlösen können, wenn sie im Rahmen entsprechender Aufklärungstechnologien auf die empirischen Einzelwissenschaften zurückgreifen; ansonsten operieren sie blind nach dem

„Backfire Effects“ bezeichnen die bereits gut untersuchten Phänomene, wenn der Versuch, Irrtümer zu korrigieren, unter bestimmten Umständen systematisch zu einer Verstärkung dieser Irrtümer führt, statt zu ihrer Reduzierung. Um diesem Effekt zu entgehen, empfehlen einige Psychologinnen, die Methoden der rationalen Diskussion und Wissensvermittlung durch indirekte Taktiken der Handlungs- und Überzeugungsänderung zu ersetzen. Hiermit gerät der ideologiekritische Anspruch jedoch in einen Konflikt mit den nötigen Mitteln zu seiner Realisierung: das Paternalismusdilemma. 
wenig erfolgsversprechenden „Prinzip Hoffnung“, also ohne die Eruierung erfolgversprechender Methoden - da die Beurteilung einer Methode als erfolgversprechend sich allein durch empirische Prüfungen erweisen kann, und nicht etwa aus einer Theorie deduziert zu werden kann.

Und zweitens kann ein derartiger Rückgriff auf die empirischen Einzelwissenschaften zu bisher wenig beachteten - aber gerade deswegen relevanten - Problemen der Aufklärungstechnologie führen. Das exemplarisch aufgeworfene Problem erzwingt sodann bestimmte bildungs- und sozialpolitische Maßnahmen zu seiner Lösung, gibt Aufklärungstechnologien also bereits einen gewissen Inhalt, der wiederum auf die Notwendigkeit bestimmten empirischen Sachwissens zu Zwecken der Ideologiekritik zurückverweist, wie im Fazit verdeutlicht wird.

\section{Begriffliche Vorklärungen}

Anfänglich sind begriffliche Konkretisierung der Begriffe „Ideologie“ und "Ideologiekritik" unerlässlich, da beide Begriffe in höchstem Maße ambig, also mehrdeutig sind. Die Ambiguität wird zwar in vielen Standardwerken erkannt - mustergültig etwa zu Beginn von Eagletons Monographie Ideologie (2000 [1993]) - doch werden selten die nötigen, anti-essentialistischen Konsequenzen daraus gezogen.

Diese Ambiguität ist in einem ersten Schritt aufzulösen - und zwar, ohne dem begriffsessentialistischen Fehlschluss aufzusitzen, die Operation mit einem „Wort" für die Identität eines „Begriffs" anzunehmen und dessen Einheit zu unterstellen. So sind etwa einige Autorinnen (bspw. Elster 1986) der Ansicht, dass sich im Werk von Marx und Engels mehrere Ideologiebegriffe ausfindig machen lassen, die nicht nur unterschiedliche Zwecke verfolgen, sondern auch nicht in einem einheitlichen Begriff aufgehoben werden können. In nicht-marxistischen Paradigmen - etwa im Neopositivismus oder im Kritischen Rationalismus - finden sich wiederum andere Ideologiebegriffe. Wenn die Ambiguität eines Begriffs derart manifest ist, muss die Klarheit des bezeichneten Gegenstands stipulativ sichergestellt werden - also durch die Festsetzung alternativer Begriffe, die die jeweiligen disparaten Konzepte "des" Ideologiebegriffs voneinander abgrenzen (Tepe 2012, 13ff; Körner 2018, 4-6). 
Ich verwende im Folgenden den Begriff „Ideologie“ ausschließlich in einem sehr formalen, weitgefassten und erkenntniskritischen Sinne: „Ideologien" sind dieser formalen Definition nach systematisch zustande gekommene epistemisch defiziente Überzeugungen. Es handelt sich also um bestimmte Überzeugungen, und zwar solche, die systematisch - und nicht etwa arbiträr - zustande kommen und epistemisch defizient, also in erkenntnismäßiger Hinsicht problematisch sind, etwa sachlich falsch oder einseitig verzerrt (Körner 2015).

Zudem sei erwähnt, dass „der“ Ideologiebegriff in fast in allen Fällen - außer vielleicht seiner positiven Identifizierung mit dem Begriff „Klassenbewusstsein", etwa in Teilen der DDR-Philosophie (die eine positive Besetzung und damit etwa Zuschreibungen wie „unsere Ideologie .... ermöglicht) - tatsächlich erkenntniskritisch ${ }^{2}$ ist: Denn es wird stets behauptet, dass zumindest die Überzeugungen zweiter Ordnung 3 der Akteure, die einer Ideologie anhängen, unzutreffend sind; im anderen Falle müssten die Akteure wissen, dass ihre Überzeugungen beispielsweise die Funktion hätten, die herrschenden Verhältnisse zu reproduzieren. Wenn die Akteure dies wüssten, würde es allerdings keiner Ideologiekritik bedürfen (Körner 2015, 8-9), da mit dem richtigen Bewusstsein eben keine Ideologie mehr vorläge.

2 Eine kritische Zurückweisung kann auf verschiedene Weisen vor sich gehen, also verschiedene Gründe für eine Zurückweisung bemühen. Doch es gibt nur eine Art solcher Zurückweisungsgründe, die beanspruchen kann, intersubjektiv gerechtfertigt und verständlich zu sein - nämlich, indem der fraglichen Theorie oder Ideologie nachgewiesen werden kann, dass sie in bestimmten Hinsichten epistemisch defizient ist. Die verbreitete zweite Art der Zurückweisung ist die, die sich auf moralisch-ethische Gründe stützt. Doch da eine strenge und intersubjektiv vernünftig nachvollziehbare Rechtfertigung moralisch-ethischer Grundsätze nicht verfügbar scheint, kann eine solche Art der Zurückweisung nur auf die letztlich arbiträre Sympathie anderer Akteure hoffen, nicht aber darauf, dass die Zurückweisung als intersubjektiv gerechtfertigt und verständlich angesehen wird. Derartige „Fronten“ zu konstruieren, erfüllt keinen guten erkenntnispraktischen Zweck, verhindert sie doch die kritische Reflexion der eigenen Position und ist willkürlich herstellbar - eine gerechtfertigte Zurückweisung muss stattdessen stets vor dem Tribunal der universellen Menschenvernunft bestehen können. Deshalb kann nur eine epistemische Kritik intersubjektiv nachvollziehbare Geltung beanspruchen (Körner 2018, 2, Fußnote 5) und ist aus diesem Grunde auch in der öffentlichen Auseinandersetzung mit politischen Ideologien unverzichtbar, da sie der eigenen Agenda die nötige intersubjektive Legitimität verleiht.

3 Überzeugungen zweiter Ordnung sind Überzeugungen über andere Überzeugungen, vor allem über ihre Genese, Geltung und Funktion. 
Der Begriff „Ideologiekritik“ wird indessen für Theorien verwendet, die einerseits näher festlegen, worin genau epistemische Defizienz besteht - in welcher Hinsicht die von ihnen als „Ideologie“ kritisierten Überzeugungen erkenntnismäßig problematisch sind - und Ideologien andererseits erklären wollen: Weshalb sie entstehen, weshalb sie persistieren und aus welchen Gründen sie welche Konsequenzen zeitigen. Die Bewältigung dieser beiden Aufgaben - der Klärung der kritisierten epistemischen Defizienz und der Erklärung der problematisierten Überzeugungen, sowie ggf. ihrer Konsequenzen - ist der theoretische Anspruch ideologiekritischer Theorien. ${ }^{4}$

Wie anfangs erwähnt besteht der praktische Anspruch der Ideologiekritik hingegen darin, Ideologien zu reduzieren, präventiv zu verhindern oder gar zu überwinden. Um diesen Anspruch erfolgreich in die Tat umzusetzen, kommen verschiedene Mittel in Frage, weshalb ich diesen Kontext den der "Aufklärungstechnologie“ nenne, also eine Technologie, die die Mittel zum Zwecke der praktischen Bekämpfung von Ideologien und ihren Ursachen zu eruieren sucht.

\section{Aufklärungstechnologischer Methodenmonismus}

Ideologiekritische Theorien setzen ihr Vertrauen in die Instrumente der Bekämpfung von Ideologien, also der Mittel ihrer expliziten oder impliziten Aufklärungstechnologie, nun leider typischerweise im Rahmen eines Methodenmonismus. Ich verwende diesen Begriff hier etwas eigenwillig und bezeichne damit das aufklärungstechnologische Programm, sich nur auf eine Art von Methode zur Bekämpfung von Ideologien zu verlassen. Typischerweise ist diese Beschränkung bereits begrifflich vorentschieden, während es doch eigentlich eine empirisch zu beantwortende Frage sein sollte, welche aufklärungstechnologischen Methoden zielführend sind (Körner 2015; Körner 2018).

4 Auch in diesem erkenntniskritischen Sinne von "Ideologiekritik“ ist es indessen unproblematisch, wenn sich einzelne ideologiekritische Theorien auf ganz bestimmte epistemisch defiziente Überzeugungen beschränken - etwa solche, die gewisse, als unethisch angesehene sozio-politische Konsequenzen zeitigen - sofern eine derartige Einschränkung des Gegenstandsbereichs transparent gemacht wird (Körner 2015, 15-16). 
So herrscht beispielsweise in der klassischen Tradition, die man auch die „aufklärerische Tradition“ von Aufklärungstechnologien nennen könnte und die sich ideengeschichtlich von Francis Bacons Idolenlehre bis zu den ideologiekritischen Ansätzen des Neopositivismus und des Kritischen Rationalismus zieht, die Ansicht vor, dass Ideologien dadurch zurückgedrängt werden, indem den Akteuren das richtige Wissen vermittelt würde - insbesondere das Wissen über diese Ideologietheorien selbst. Hinter diesem Vertrauen in die aufklärende Kraft des relevanten Sachwissens steckt die Idee, dass der Bann „falscher Ideen“ gebrochen würde, wenn man von ihnen, ihrer Entstehung, ihrer Funktion und ihrem Verfestigungsmechanismus Kunde hat. Andere Methoden außer dieser Vermittlung ideologiekritischer Bildung werden typischerweise entweder nicht in Betracht gezogen oder haben nur eine andienende Funktion für die Primärmethode der Bildungsvermittlung. Der Kürze halber wird diese Methode im Folgenden als „ideelle Aufklärung“ bezeichnet.

Das andere große ideologiekritische Paradigma zur programmatischen Gestaltung von Aufklärungstechnologien findet sich vor allem in der Tradition des Marxismus. ${ }^{5}$ Die marxistische Ideologiekritik kritisiert unter anderem die - ihrer Theorie nach - falsche Vorstellung der "Selbstständigkeit" der Ideen vor dem Hintergrund ihrer praktischen Veränderung: Denn wenn die marxistische Ideologiekritik wahr ist, kann der klassisch aufklärerische Anspruch, epistemisch defizientes Denken durch die Vermittlung zutreffender Theorien zurückzudrängen, nicht eingelöst werden. Stattdessen sei eine Änderung der sozio-ökonomischen Bedingungen erforderlich, um das Denken und damit die Überzeugungen der Akteure zu ändern. Die junghegelianischen Religionskritiker gelten Marx eben deshalb als "Ideologen“, da sie "die revolutionäre Veränderung des Bestehenden unterlaufen, indem sie diese als Bewußtseinsveränderung interpretierten" (Bohlender 2010, 44, kursiv von PK). Diese Erkenntniskritik an Überzeugungen zweiter Ordnung bestimmt den zentralen Charakter des Ideologischen in dem systematischen Irrtum, dass das Denken nicht durch äuBere Bedingungen präformiert und bedingt sei. Das Denken wird also irrtümlicherweise als unabhängig - und damit von den konkreten Lebensbedingungen getrennt - verstanden.

5 Der Fokus liegt hier und im Folgenden auf dem Konzept in der Deutschen Ideologie (Marx und Engels 1978 [1958]). 
Die Pointe der frühen Ideologiekritik Marxens zeigt sich vor allem in ihrer Anwendung auf den Gegenstand der Aufklärungstechnologie - denn Marx wirft den Philosophen vor, durch ihre rein theoretisch bleibende Kritik und ihren klassisch aufklärerischen Anspruch, die Welt zu verbessern, indem sie unsere Interpretationen von der Welt verbessern, völlig fruchtlos zu bleiben und auf diese Weise sogar den Status quo der herrschenden Verhältnisse zu zementieren, während eine recht verstandene Kritik nach Marx nur in einer praktischen Änderung des Bestehenden Erfolge zeitigen und durch diese Veränderung des Bestehenden auch erst das Denken ändern könne. Im Folgenden wird dieses auf die Veränderung der Lebensumstände der aufzuklärenden Akteure abzielende ideologiekritische Programm als „materielle Aufklärung“ bezeichnet.

Marxens Pointe beruht allerdings auf einer Konfundierung ideologiekritischer Analyse mit Strategien der Aufklärungstechnologie. Es folgen zwei Beispiele für die logische Unabhängigkeit aufklärungstechnologischer Methoden von ideologiekritischen Analysen.

Selbst dann, wenn die These vom Primat der sozio-ökonomischen Bedingungen als zutreffend angesehen wird, müsste die Persistenz der Ursachen - also konkreter sozio-ökonomischer Bedingungen - nicht zwangsläufig die Unüberwindbarkeit ihrer Effekte implizieren, solange diese Ursachen selbst nicht ausgeräumt sind. Es könnte sich prinzipiell auch so verhalten, dass trotz Persistenz der Ursachen ihre Symptome auf anderen Feldern erfolgreich bekämpft werden können - so wie eine Impfung auch dann gegen Krankheitserreger schützt, wenn Letztere weiterhin wirksam sind. Beispielhaft für eine derartige Strategie wäre das bereits erwähnte aufklärungsphilosophische und auch in der modernen Psychologie manifeste Vertrauen, dass die Kenntnis der Wirkungsweise erkenntnisverzerrender Faktoren auch deren Effekte zu kontrollieren helfe.

Ebenso ist es möglich, dass die einmal generierten Effekte sozio-ökonomischer Bedingungen eine Art Eigenleben führen, in kulturelle Traditionen und Institutionen Einzug finden oder sich auf andere Weise verselbstständigen, ${ }^{6}$ sodass die Änderung sozio-ökonomischer Bedingungen nicht auch eine Änderung ihrer Effekte mit sich bringen muss. ${ }^{7}$

6 Hierfür müsste eine gewisse Eigendynamik des „Überbaus“ im Sinn der marxschen Theorie anerkannt werden, was Marx ja auch später tatsächlich konzediert.

7 Eine derartige Verselbstständigung ist auch auf der Ebene begründungsfundierter Überzeugungen zu beobachten: „Psychologische Experimente haben eindrucksvoll 
Die materielle Aufklärung setzt also Ätiologie und Therapie vorschnell gleich: Demnach könne die Behebung der Effekte, die eine Ursache hervorbringt, allein durch die Behebung dieser Ursache zustande gebracht werden. In Analogie etwa zur Psychoanalyse erscheint es zwar plausibel, dass eine Symptombekämpfung ohne Ursachenbekämpfung nicht sonderlich zielführend sein dürfte (da sich die Ursache dann in anderer Gestalt dennoch geltend machen würde) - es ist allerdings eine empirische Frage, ob es sich im jeweiligen Falle wirklich so verhält. Dennoch geht materielle Aufklärung apodiktisch davon aus, dass Ideologien dann und nur dann absterben, wenn ihre Ursachen beseitigt werden - und wenn man sich den politischen Kontext der marxistischen Ideologiekritik vor Augen führt, ist das Festhalten an diesem vorschnellen Schluss auch sehr naheliegend, da es sich um eine Theorie handelt, die zu praktisch-politischer Veränderung drängt und diese zu legitimieren versucht. ${ }^{8}$

Gegen die Engführung materieller Aufklärung, laut der die Bekämpfung von Ideologien nur und ausschließlich durch die Bekämpfung ihrer sozio-ökonomischen Ursachen erfolgreich sein kann, können wir also zumindest skizzenhaft folgende Alternative stellen: Auch wenn wir die Genese und Adaption unserer Überzeugungen kaum kontrollieren können, so doch in größerem Ausmaß die Art, wie wir post hoc mit ihnen verfahren. Wir können uns selbst auf unsere Überzeugungen und deren Vernünftigkeit hin befragen, wir können uns - ggf. mit Hilfe von Anderen - selbst kritisieren, uns von unseren Überzeugungen lösen oder sie schärfen und veredeln.

gezeigt, dass Menschen in der Tat dazu neigen, an Überzeugungen auch dann festzuhalten, wenn die ursprünglichen Rechtfertiger verschwinden und keine neue[n] Evidenzen an ihre Stelle treten." (Baumann 2006, 186)

$8 \quad$ Hinter dem Programm sowohl der ideellen wie der materiellen Aufklärung steckt freilich eine in der klassischen Philosophie populäre Idee, und zwar, dass Irrtümer und andere epistemisch defiziente Überzeugungen ausschließlich durch Faktoren bedingt seien, die gewissermaßen außerhalb der Vernunft und ihrer angemessenen Anwendung lägen - etwa Wünsche und Bedürfnisse oder aber eben sozio-ökonomische Bedingungen. Erst in der Aufklärungsphilosophie dämmerte eine Ahnung, die schließlich von der empirischen Psychologie bestätigt wurde: Unsere Vernunft ist nicht so "rein" und autonom, wie erhofft, sondern einige Faktoren für Verzerrungseffekte sind in unserem kognitiven Apparat angelegt. Diesen Einsichten Rechnung zu tragen hieße deshalb auch, Erkenntniskritik nicht allein über die Konzentration auf vernunft-externe Faktoren betreiben zu können. Auf die somit problematisch gewordene Selbstüberschätzung der Vernunft sei im Folgenden noch zurückzukommen. 
Die Tradition der materiellen Aufklärung hat auch in dieser Hinsicht zu viel Gewicht auf das Zustandekommen defizienter Überzeugungen gelegt - doch die intellektuelle Arbeit mit und an unseren Überzeugungen, die wir tagtäglich beobachten können, hat keine Konsequenz für dieses Aufklärungsprogramm gezeitigt, obwohl eine solche Berücksichtigung vor allem für Philosophinnen und andere Intellektuelle performativ nahe liegen dürfte: Wir können nicht widerspruchslos denken, dass unsere Theorien und deren Beurteilung nicht zumindest auch durch vernünftiges Räsonieren, sondern allein durch unsere sozio-ökonomischen Umstände bestimmt werden (Körner 2018, 7-12). Wenn wir materielle Aufklärung ernst nähmen, könnten wir dieses Aufklärungsprogramm selbst kaum zufriedenstellend erklären: Auf die Frage, weshalb es jemand als plausibel ansieht und sich um eine dementsprechende ideologiekritische Verbesserung der Welt bemüht, müsste auf Einsicht und Argumente, die eigene Bildungsbiographie, das Lesen einschlägiger Texte oder die Diskussion mit Kolleginnen oder Genossinnen verwiesen werden - womit bereits praktisch plausibilisiert wäre, dass selbst im Rahmen eines materiellen Aufklärungsprogramms auch ideelle Aufklärung qua Bildungsvermittlung berücksichtigt werden muss.

Diesem Instrument der Bildungsvermittlung zur Bekämpfung von Ideologien werde ich mich nun zuwenden.

\section{Selbstbefreiung durch das Wissen}

Traditionell wird das Ziel der Aufklärung darin gesehen, die Unmündigkeit des Menschen zu reduzieren und seine Autonomie zu forcieren - was durch Selbstdenken, die Emanzipation von Autoritäten und zutreffendes Sachwissen geleistet werden soll. Aus diesem Grunde sollten Aberglauben, Vorurteile, Irrtümer und illegitime Rechtfertigungen sozialer Ordnungen bekämpft werden, die den Menschen im Banne halten. Karl R. Popper brachte diese Agenda durch das Ideal der „Selbstbefreiung durch 
das Wissen“ auf den Begriff. ${ }^{9}$ Doch der Begriff lässt unbestimmt, welche Arten von Wissen für die Realisierung dieses Ideals in Frage kommen. ${ }^{10}$

Bevor ich mich dieser Frage zuwende, ist eine kurze Anmerkung nötig: Es muss betont werden, dass das aufklärerische Programm der "Selbstbefreiung durch das Wissen“ über den praktischen Anspruch der Ideologiekritik hinaus auch noch ein weiteres Ziel verfolgt, und zwar das gerade bereits genannte Ziel, die Autonomie der aufzuklärenden Akteure - und damit auch ihre epistemische wie politische Mündigkeit - zu erhöhen (Gröbl-Steinbach Schuster 2011, 180). Auch wenn eine Förderung individueller Autonomie meist als implizites Ziel jeder Ideologiekritik genannt wird, wäre es logisch möglich, sowohl einerseits bestimmte epistemisch defiziente Überzeugungen zu bekämpfen als auch andererseits auf das zusätzliche Ziel der Förderung der Autonomie zu verzichten. Doch das Aufklärungsprogramm der "Selbstbefreiung durch das Wissen“ zielt nicht nur auf den aufklärungstechnologisch vermittelten Kampf gegen Ideologien ab, sondern beabsichtigt dadurch zugleich die Autonomie der betroffenen Akteure zu erhöhen. Die Voraussetzung dieser charakteristischen Zielkonstellation führt allerdings schließlich zu dem noch zu thematisierenden Paternalismusdilemma.

Die Förderung der Autonomie soll dem Menschen im Rahmen eines egalitären Erkenntnisprogramms zum Status eines mündigen Subjekts verhelfen, das die Freiheit in der Verfolgung eigener Ziele besitzt, was impliziert, mögliche und faktische Beschränkungen dieser Freiheit angemessen erkennen und kontrollieren zu können, wozu externe Faktoren - etwa die Autorität des Klerus - und interne Faktoren - wie Wunschdenken in den idola tribus Francis Bacons - gehören, die die eigenen Überzeugungen

9 „So enthielt die Idee der Selbstbefreiung durch das Wissen, die die Aufklärung vertrat, von Anfang an auch die Idee, dass wir lernen müssen, uns von unseren eigenen Ideen zu distanzieren, statt uns mit unseren Ideen zu identifizieren. Die Erkenntnis von der geistigen Macht der Ideen führt zu der Aufgabe, uns von der geistigen Übermacht falscher Ideen zu befreien. Im Interesse der Wahrheitssuche und der Befreiung vom Irrtum müssen wir uns dazu erziehen, unsere eigenen Ideen ebenso kritisch betrachten zu können wie die Ideen, gegen die wir kämpfen." (Popper 2006 [1987], 162)

10 Anbei sei der Vorwurf der historischen Relativität unseres „Wissens“ zu entkräften: Selbst dann, wenn sich das verwendete Sachwissen als falsch herausstellen mag, so sensibilisiert uns die methodische Herangehensweise, die unsere Überzeugungen zum Objekt kritischer Beurteilung macht und ihre mögliche Falschheit anerkennt, für unsere Rechtfertigungspraxis und mögliche Fehlstellungen, die dabei auftreten können. 
präformieren. ${ }^{11}$ Diese Faktoren könnten - so das aufklärerische Motiv kontrolliert und somit abgestellt werden, wenn sie erkannt würden. Dies macht es nötig, die Menschen über jene Faktoren zu belehren und die Lehre so zu formulieren, dass sie ihr ohne Rückgriff auf Autoritätsargumente Folge leisten können.

Hiermit ist auch bereits eine Antwort auf die Frage nach dem nötigen Sachwissen gefunden, das eine "Selbstbefreiung" anleiten kann: Gefordert ist nicht einfach jedes beliebige Wissen über die Erfahrungswelt, sondern vor allem ein Wissen über unsere Erkenntnis- und Rechtfertigungspraxis: über die Ursachen unserer Überzeugungen und damit verbundene Gründe für Irrtümer und Verzerrungen. Wir benötigen also Sachwissen über die Unvollständigkeit unserer Rationalität (Elster 1987) - und dies ist ein Wissen, das wir vor allem aus den akademischen Disziplinen der Psychologie und Soziologie kennen - man denke etwa an die Studien zum autoritären Charakter von Milgram und Zimbardo.

Mit Sachwissen über die Unvollständigkeit unserer Rationalität sollen zwei Formen der Korrektur Hand in Hand gehen: einerseits die Korrektur von Irrtümern, die dem Akteur zuvor nicht als Irrtümer erschienen; und andererseits über Gründe und Ursachen für diese Irrtümer.

Da es im Speziellen um fehlerhafte Arten des Räsonierens geht und sich dies erst vor der Kontrastfolie vernünftigen Räsonierens zeigt, sind für das von der ideellen Aufklärung angestrebte Ziel auch Theoreme aus der Erkenntnis- und Wissenschaftstheorie nötig. ${ }^{12}$ Denn erst das Wissen aus

11 Es ist zu beachten, dass Sachwissen die Anzahl von Handlungsalternativen nicht per se erhöht, wohl jedoch die Zielerfüllung unserer Handlungen optimiert. Dass Sachwissen auch zu einer Beschränkung von Handlungsalternativen führen kann, verdeutlichen Beispiele über das Wissen hinsichtlich unerwünschter Konsequenzen und Implikationen unserer Handlungen, die herausstellen, dass zuvor als unproblematisch angesehene Handlungen de facto im Konflikt mit bestimmten Werten oder Überzeugungen stehen - wie es etwa beim Wissen über ökologische Konsequenzen des Personenflugverkehrs oder des Kaufes tierischer Produkte der Fall ist.

Die Idee hinter der aufklärungstechnologischen Relevanz der philosophischen Erkenntnis- und Wissenschaftstheorie basiert auf der formalen, erkenntnistheoretischen Trennung von Geltung und Genese einer Überzeugung unter der Vermeidung genetischer Fehlschlüsse: Allein durch die Genese einer Überzeugung lässt sich noch kein Urteil über die Geltung jener Überzeugung rechtfertigen - Letztere müsse unter der Voraussetzung formaler Strenge eigenständig beurteilt werden. Die durch die empirischen Einzelwissenschaften vermittelte Kenntnis der Genese problematischer Überzeugungen kann allein der Heuristik des Verdachts dienen. Doch Autonomie hin- 
diesen philosophischen Disziplinen erlaubt eine methodische Kritik, die auch die Kritik an den eigenen Überzeugungen anzuleiten vermag. Und erst mit diesem Wissen ist es möglich, vernünftig zu entscheiden, ob eine Theorie oder Überzeugung als glaubwürdig oder unglaubwürdig angesehen werden muss: Soll etwa entschieden werden, ob eine marxistische Erklärung eines bestimmten Phänomens, oder die konkurrierende Erklärung, die sich auf verschwörungstheoretische Überlegungen stützt, bevorzugt werden soll, ist der Rückgriff auf Erkenntnis- und Wissenschaftstheorie, sowie die Berücksichtigung eigener Affinitäten für kognitive Fehlleistungen unabdingbar. ${ }^{13}$

Zusammengenommen ließe sich sagen: Kritisches Denken setzt spezifisches Sachwissen über kritisches wie unkritisches Denken voraus. ${ }^{14}$

Die ideologiekritische Emanzipation der Vernunft muss nun das Wissen um die Grenzen und Schwächen der Vernunft reflektiert integrieren, denn sonst gibt sie sich - analog zu anderen szientistischen Kontexten, also solchen, in denen wissenschaftliche Leistungsfähigkeit überschätzt wird - systematisch ein Zerrbild ihrer selbst und behindert damit praktische Emanzipation, also die Erfüllung ihres Ziels. Eine autonome Selbstkontrolle ist erst dann möglich, wenn die Potentiale eigener Fehlleistungen bekannt sind - und deshalb benötigt man Wissen über die unvoll-

sichtlich Erkenntnisfragen muss die Kompetenz implizieren, eigenständig die Geltung von Überzeugungen und die Vernünftigkeit ihrer Adaption beurteilen zu können.

Körner (2017) zeigt dies exemplarisch für die Beurteilung von Verschwörungstheorien.

Dieses Ziel sei auch der Grund für die von den französischen Aufklärungsphilosophen forcierte Enzyklopädie gewesen: „Möglichst viele Menschen sollten in die Lage versetzt werden, sich durch Aneignung von Sachwissen von vielfältigen Abhängigkeiten, sei es von eingebildeten Naturnotwendigkeiten oder unnötigen politisch-sozialen Zwängen, frei zu machen." (Salamun 1991, 96) Während Salamun diese aufklärerische Tradition affirmiert, differenziert er allerdings kaum zwischen dem Sachwissen per se, das Gegenstand der Enzyklopädie war, und den in seiner eigenen ideologiekritischen Konzeption aufklärungstechnologisch relevanten Kenntnisständen - nämlich vor allem Methoden, mittels derer rationales Problemlösungsverhalten und das Bilden selbstständiger Urteile ermöglicht werden sollen, um gegen jede Form dogmatischer und verschleiernder Einflussstrategien - Leerformeln, Obskurantismus, Wertaussagen mit dem Anstrich von Sachaussagen - vorzuschützen (ebenda, 115). Eine derart undifferenzierte Perspektive auf Sachwissen und die Förderung von Autonomie und Mündigkeit ist indessen recht typisch für Literatur zu den Themen Aufklärung und Ideologiekritik. 
ständige Rationalität, also ein Wissen, das auf der Ebene der Überzeugungen zweiter Ordnung angesiedelt ist.

Eine Ideologiekritik, die dies berücksichtigt, wird den Fehler der klassischen Aufklärungsphilosophie tendenziell vermeiden können, die Autonomie des richtigen Denkens überoptimistisch im Sinne einer Selbstüberschätzung der Vernunft zu besetzen. Die Anerkennung unvollständiger Rationalität und der Grenzen vernünftigen Räsonierens erzwingt nicht nur eine Anpassung der Zielstellung, Adressatenperspektive und Aufklärungstechnologie von Ideologiekritik, sondern auch die undogmatische Offenheit ihrer eigenen Konzeption - Ideologiekritik als Theorie und Praxis darf das Potential eigener Fehlleistungen nicht unterschlagen. Sowohl auf der Adressaten- als auch auf der Theorieebene wird diese Einsicht noch durch die Anerkennung der Kontextabhängigkeit unseres Denkens untermauert, über die Ideologiekritik mittels adäquater Überzeugungen zweiter Ordnung zwar eine gewisse Kontrolle gewinnen - und damit die Spielräume eigener Autonomie teilweise erweitern -, sie aber kaum vollends ausschalten kann.

Jon Elster verdeutlicht dies sehr anschaulich am Beispiel der Geschichte von Odysseus und den Sirenen aus der Odyssee: Odysseus weiß durch Kirke um seine Schwäche - die Unfähigkeit, sich kontrollieren zu können, sobald er in die Reichweite des Sirenengesangs geraten wird. Nur mit Hilfe dieses Wissens kann er Methoden einsetzen, um der Realisierung seiner Schwäche prospektiv vorzubeugen, was den Spielraum seiner Möglichkeiten erhöht, autonom mit seinen - abzusehenden - Bedürfnissen, Gedanken und Emotionen zu agieren. Kirkes Informationen spielen hier die Rolle idealiter zutreffenden Sachwissens über die unvollständige Rationalität, womit überhaupt erst Selbstkontrolle ermöglicht wird: Wenn wir von diesen Beschränkungen wissen, so können wir sie zu kontrollieren versuchen (Elster 1987, 67ff.). Sachwissen kann also selbst dann aufklärerisch wirksam sein, wenn man einen überoptimistischen doxastischen Voluntarismus ablehnt, demzufolge wir unsere Überzeugungen willentlich kontrollieren könnten: ${ }^{15}$ "Auch wenn der Mensch nicht rational ist,

Statt sich der Illusion einer völligen Beherrschung unserer kognitiven Mechanismen hinzugeben, können wir mit dem adäquaten theoretischen Rüstzeug eine Selbstkontrollinstanz installieren und auf diese Weise methodisch Vorsicht vor unserem eigenen Denken und Handeln walten lassen. So kann etwa in psychologischen Experimenten die Bekanntgabe der Forschungshypothese zu einer Angleichung der (Leistungen der) Versuchs- und Kontrollgruppe führen, wie an Nachfolgeexperimenten der Milgram- 
weiß er, dass er irrational ist, und kann sich selbst binden, um sich vor der Irrationalität zu schützen" (ebenda, 140).

Die Abhängigkeiten, die der Selbstbefreiung im Wege stehen, sind also nicht nur externer Art - etwa durch Autoritäten und Konformitätsdruck - sondern auch interner Art, etwa in der Form von Befangenheiten, Affekten und fehlerhaften Methoden des Denkens. Um sich von diesen Abhängigkeiten so weit wie möglich zu befreien, ist es in jedem Falle nötig, eine kritische Distanz zu den eigenen Überzeugungen zu entwickeln, statt sich mit diesen zu identifizieren - nur so können die eigenen Überzeugungen kritisch objektiviert und kann die potentielle Falschheit eigener Überzeugungen praktisch anerkannt werden; erst dann wird methodische Selbstkritik ermöglicht und ist es prinzipiell opportun, Selbstkritik zu praktizieren und zuzulassen. Die Etablierung einer kritischen Distanz zu den eigenen Ideen ist somit die Institutionalisierung eines Korrektivs gegen eigene Fehleranfälligkeiten, wie sie sich auch in Techniken der "Selbstbindung" am Beispiel Odysseus niederschlägt.

Das Programm einer solchen ideellen Aufklärung scheint also unabhängig von einem speziellen Zuschnitt auf die Ausräumung von Irrtümern zu sein. Dann wäre sie identisch mit der Vermittlung wissenschaftlicher Praktiken und Ergebnisse zu bestimmten, nämlich aufklärungstechnologisch relevanten Themenfeldern, etwa der Psychologie, Soziologie, Erkenntnis- und Wissenschaftstheorie, ihren Theorien und Methoden, sowie historischer Bildung gegen die Tendenz zur Naturalisierung des Status quo. Man sieht also en passant, dass Wissenschaften auch ohne normativen Zuschnitt oder eine ausgewiesen „kritische Methode", sondern allein durch die Konzentration auf bestimmte Forschungsgegenstände und ihre angemessene Vermittlung kritisch sein können. ${ }^{16}$

Experimente gezeigt wurde: Wenn Probanden die kausale Forschungshypothese mitgeteilt wurde, so handelten die Akteure anders als prognostiziert, da sie nun über ihre kognitiven Mechanismen bei Kontakt mit dem Experiment aufgeklärt waren und es somit nicht zu einer „Kausalität aus Unkenntnis“ kam (Keutner 2004, 223).

16 Emanzipatorische Kritik benötigt keine normativen Aussagen und keine gesonderte Methode, sondern die normalwissenschaftliche Analyse emanzipationsrelevanter Untersuchungsgegenstände. Auch eine werturteilsfreie Analyse kann falsche Vorstellungen und Verschleierungen realer Tatbestände durchsichtig machen - besonders deutlich etwa in der aufklärerischen Kritik des Gottesgnadentums als politischem Legitimationsinstrument - und die Akteure durch dieses Wissen befähigen, sich von entsprechenden Abhängigkeiten zu befreien (Albert 1960, 229ff). Am Rande sei allerdings erwähnt, dass die Wissenschaften hierfür die Freiheit haben müssen, ihre Forschungs- 
Allerdings muss hier eine Brücke zur materiellen Aufklärung geschlagen werden: Ob tatsächlich Aufklärung aus der Wissens- und Methodenvermittlung folgt, hängt ab von der praktischen Binnenrationalität der Akteure, die bestimmt wird von ihren Umständen, objektiven Sachzwängen und Ressourcenproblemen. Wiegen bestimmte Lebensinteressen schwerer, mögen die Akteure zwar grundsätzlich im Besitz der aufklärungsrelevanten Bildung sein, doch ohne dass diese zur Anwendung kommt womit ihr praktisch-politisches Ziel unerfüllt bliebe.

Die reine Bereitstellung von spezifischer Bildung reicht also nicht aus - hinzu muss auch die praktische Freiheit samt Ressourcen treten, die es erst binnenrational machen, sich um die aufwändige selbstkritische Emanzipation hin zur Mündigkeit zu bemühen. ${ }^{17}$

\section{Paternalismusdilemma I}

Trotz der Rede von der "Selbstbefreiung“ dürfte offensichtlich sein, dass das skizzierte Programm einer "aufklärerischen“ Aufklärungstechnologie eine Fremd-Aufklärung darstellt - denn die aufzuklärenden Akteure verfügen noch nicht über die kritischen Kompetenzen, um die Legitimität der Adaption des gerade genannten Wissens eigenständig beurteilen zu können. Diese kritischen Kompetenzen sollen ja überhaupt erst gefördert werden. Dementsprechend können jene kritischen Kompetenzen bei der Beurteilung jener kritischen Kompetenzen selbst nicht vorausgesetzt werden. Es ist hierbei auch unerheblich, ob die zu vermittelnde Bildung sich in erster Linie durch klassisches Sachwissen - "knowing that" - oder durch Methodenwissen - "knowing how“ - charakterisiert, da Letzteres ebenfalls Sachwissen, wenn auch in technologischer Formulierung, dar-

gegenstände selbstständig und ohne den Druck externer, vor allem emanzipationsfeindlicher Interessen wählen zu können.

17 Einem derartigen Konzept von Binnenrationalität, das den Fall zulässt, dass Akteure rational zu epistemisch defizienten Überzeugungen und daran anschließenden Handlungen gelangen, liegt indessen eine Differenzierung "epistemischer" und "prudentieller Rationalität“ zugrunde (vgl. etwa Gosepath 1992). Im Gegensatz zu Gosepath wird hier zudem von der Voraussetzung ausgegangen, dass eine Überzeugung zugleich prudentiell rational und epistemisch irrational sein kann, dass also lakonisch gesagt Lebensinteressen für Akteure mitunter gegenüber Erkenntnisinteressen prioritär sind (vgl. dazu auch etwa Boudon 1988 und Schmid 2004). 
stellt. In jedem Falle müssten die aufzuklärenden Akteure allerdings einen „blinden Glauben“ an den emanzipatorischen Nutzen der fraglichen Bildung an den Tag legen respektive durch Manipulationsmethoden dazu gebracht werden, diese als gültig anzunehmen.

Insofern ist es vielleicht verständlich, dass auch schon der historischen Aufklärungsphilosophie unterstellt wurde, paternalistisch zu sein, also die „aufzuklärenden“ Akteure zu bevormunden, während sie doch mit dem noblen Anspruch auftrat, zur Mündigkeit zu erziehen. Dieser vermeintliche Widerspruch wird im Folgenden aufgegriffen.

\section{Die Relevanz empirischen Sachwissens am Beispiel von „Backfire Effects"}

Nachdem die Notwendigkeit ideeller Aufklärung, ihre Inhalte und Grenzen skizziert wurden, wird nun am Beispiel der in der Psychologie untersuchten "Backfire Effects" die Relevanz der empirischen Einzelwissenschaften für die Beurteilung der Erfolgsbedingungen aufklärungstechnologischer Methoden angezeigt.

„Backfire Effects“ (oder auf Deutsch: „Bumerang-Effekte“) bezeichnet das Phänomen, dass Akteure, deren fehlerhafte Überzeugungen durch zutreffenderes Sachwissen korrigiert werden sollen, mitunter dazu neigen, ihre entsprechenden Überzeugungen nicht nur nicht angemessen zu modifizieren, sondern aufgrund des Überzeugungsversuchs mit noch größerem Eifer zu vertreten.18 Diese Effekte betreffen also das Problem der Erklärung der Persistenz fehlerhafter Überzeugungen, das in der Geschichte ideologiekritischer Theorien immer wieder diskutiert wurde und teilweise für die Fruchtlosigkeit ideeller Aufklärungstechnologie verantwortlich gemacht wurde - denn diese Effekte können verhindern, dass die Vermittlung von Sachwissen die erhofften Resultate zeitigt.

So konnte etwa in Studien mit experimentellem Versuchsaufbau nachgewiesen werden, dass nur 2\% einer Gruppe von Akteuren, deren fehlerhafte Überzeugung korrigiert werden sollte, tatsächlich ihre Über-

18 "However, individuals who receive unwelcome information may not simply resist challenges to their views. Instead, they may come to support their original opinion even more strongly - what we call a ,backfire effect'." (Nyhan und Reifler 2010,9) 
zeugung änderten, während 32\% der zuvor noch unsicheren Probanden nach dem Korrekturversuch auf die fehlerhafte Überzeugung einschwenkten. Das Resultat dieser Studien war eindeutig: Der Korrekturversuch affirmiert die zu korrigierende Überzeugung.

Eine mögliche Ursache für „Backfire Effects“ sehen die Psychologen Nyhan und Reifler (2010) in folgendem Mechanismus: Die Akteure, die mit konkurrierenden Überzeugungen konfrontiert werden, reagieren argumentativ auf diese Gegenposition. Sie reaktivieren vorhandene Argumente oder suchen nach neuen. In beiden Fällen jedoch sind ihnen ihre eigenen Argumente nun präsenter, als sie es vor der Konfrontation mit der Gegenposition waren. Im Verlaufe der Argumentation und danach liegen ihnen im Spektrum ihrer Aufmerksamkeit mehr Argumente für die eigene Position vor, als es zuvor der Fall war, denn der Akteur denkt primär darüber nach, wie er seine Position verteidigen kann. So erklären Nyhan und Reifler, dass bei einer Studie zur Irrtumskorrektur mit Wählern der Republikanischen Partei in den Vereinigten Staaten 32\% der Konservativen vor dem Korrekturversuch ihre Zustimmung $\mathrm{zu}$ dem fraglichen Thema bekundeten, aber $64 \%$ von ihnen nach dem Korrekturversuch; nur $2 \%$ der beteiligten Konservativen änderten tatsächlich ihre Überzeugung. 19

Aufgrund der experimentellen Bedingungen konnten allerdings die Variablen verändert werden, sodass einige aufklärungstechnologisch interessante Erkenntnisse über diejenigen Faktoren gewonnen werden konnten, die „Backfire Effects“ begünstigen: Diese Effekte treten etwa umso häufiger und stärker auf, je häufiger die fehlerhafte Ausgangsüberzeugung erwähnt wird, je mehr widersprechende Informationen und Argumente genannt werden, je radikaler sich die „richtigere“ Alternative von der Ausgangsüberzeugung unterscheidet und je mehr auf Textkommunikation anstatt auf Grafiken zurückgegriffen wird (Cook und Lewandowsky 2012 [2011]).

Mittels der Erklärung dieser Wirkungszusammenzusammenhänge und der Isolierung und Modifikation der Variablen konnte in experimentellen Anschlussuntersuchungen herausgearbeitet werden, welche Merkmale die Wahrscheinlichkeit von „Backfire Effects“ beeinflussen: Cook nach dem Korrekturversuch - hier lag also kein „Backfire Effect“ vor. 
und Lewandowsky (2012 [2011]) nennen vor allem Vertrautheit, die durch Wiederholung erzeugt wird und die Wahrscheinlichkeit erhöht, dass eine Überzeugung als wahr angesehen wird; Informationsüberladung, die zu Überforderung und einem Rückzug auf die Ursprungsüberzeugung führt; und das Maß an Abweichung von der gefestigten Weltanschauung der Probanden, das bei gravierender Abweichung Verteidigungsstrategien provoziert.

Derartige exemplarische empirische Erkenntnisse können also nicht nur von Nutzen sein, um die Schwächen aufklärungstechnologischer Methoden ausfindig zu machen, sondern etwa durch experimentell kontrollierbare Faktorenanalysen zugleich Hilfestellungen dabei geben, aufklärungstechnologische Methoden zu verbessern. Sie sind also in besonderem Maße geeignet, die Erfolgsbedingungen ideologiekritischer Praxis zu beurteilen. Zudem erlauben sie - ebenfalls aufgrund ihrer empirisch-experimentellen Vorgehensweise - die Identifizierung unerwarteter Faktoren und Konsequenzen aufklärungstechnologischer Bemühungen; sie sind also in höchstem Maße lehrreich, insbesondere hinsichtlich ansonsten unentdeckter Schwächen ideologiekritischer Praxis.

Bei derartigen Verbesserungsversuchen können nun allerdings auch unerwartete Probleme auftreten.

\section{Paternalismusdilemma II}

Die Erkenntnisse über „Backfire Effects“ und ihre Stärke sind derart gravierend, dass einige Psychologinnen daraus den frustrierten Schluss zogen, dass Versuche der Irrtumskorrektur von vornherein zum Scheitern verurteilt seien: So führen Taber und Lodge $(2006,767)$ in diesem Rahmen an, dass ,ignorance and apathy” der fruchtbarste Nährboden für die Korrektur von Irrtümern seien, und folgern zynisch: „Perhaps we have been looking for rational citizenship in all the wrong places, and it is the great unwashed who save democracy!" (Ebenda) Andere, etwa Cook und Lewandowsky (2012 [2011], 4), empfehlen aufgrund analoger Ergebnisse, sich hinsichtlich der Bemühungen um die Korrektur von Irrtümern nur noch auf die noch nicht weltanschaulich gefestigte „Mitte“ zu konzentrieren, die „unentschlossene Mehrheit".

In den psychologischen Studien zu „Backfire Effects“ werden nun mitunter zahlreiche Methoden präsentiert, die dabei hilfreich sein sollen, 
„Backfire Effects“ zu vermeiden. Ich möchte hier nur wenige dieser Methoden knapp benennen: Erstens sollte die fehlerhafte Überzeugung weniger prominent und v.a. seltener erwähnt werden, stattdessen verstärkt die Gegenargumente (Cook und Lewandowsky 2012 [2011], 2). Zweitens sollten wenige, dafür zentrale Gegenargumente und der Anfangsüberzeugung widersprechende Informationen in möglichst einfacher Form und bestenfalls grafisch statt textlich präsentiert werden. ${ }^{20}$ Drittens sollte das positive Selbstwertgefühl der Akteure vor und während des Korrekturversuchs erhöht werden - so sollten Probanden etwa vor dem Korrekturversuch einen kurzen Text zu Situationen schreiben, in denen sie sich in der letzten Zeit besonders ehrenhaft verhalten hätten. ${ }^{21}$ Diese Strategie erweist sich als besonders zielführend bei der Vermeidung von „Backfire Effects“. Viertens sollte die Akzeptanz für die Korrektur dadurch erhöht werden, semantische Trigger $\mathrm{zu}$ vermeiden: So reagieren USamerikanische Republikaner deutlich aufgeschlossener gegenüber dem Wort "Abgaben" als gegenüber dem hochgradig negativ assoziierten Wort „Steuern“ (Cook und Lewandowsky 2012 [2011], 4). Die fünfte und letzte Strategie besteht darin, auf die Korrektur von Überzeugungen zu verzichten, da sich die "Backfire-Effects“ als zu manifestes Problem herausgestellt haben, und stattdessen Techniken des "Nudging“ zu verwenden, um das Verhalten der Akteure zu ändern - beispielsweise wird empfohlen, darauf zu verzichten, die Überzeugung zu forcieren, dass Fahrzeuge klimaschädlich seien, sondern das Verhalten etwa durch entsprechende Steuern zu regulieren:

20 In einer Anschlussstudie konnte gezeigt werden, dass die Tendenz, bei einer fehlerhaften Überzeugung zu verharren, signifikant durch die Wahl des Informationsmediums reduziert werden kann: Die Wahrscheinlichkeit der Beibehaltung der falschen Anfangsüberzeugung sank von $56 \%$ auf $33 \%$, wenn Bilder anstatt Texte für die Kommunikation der Gegenargumente verwendet wurden (Nyhan und Reifler 2017).

21 Die zweite Strategie, die Nyhan und Reifler (2017) testeten, beruhte auf der Hypothese, die Probanden würden mittels „Backfire Effects“ reagieren, weil sie durch die konkurrierende Überzeugung ihre Selbst-Integrität in Frage gestellt sahen - die Forscher vermuteten in „Backfire Effects“ einen Mechanismus zur Reduzierung kognitiver Dissonanz. Das Selbstwertgefühl der Probanden zu stärken würde, so die Hypothese, die gefühlten mentalen Kosten für die Aufgabe der Anfangsüberzeugung senken. Tatsächlich sank die Wahrscheinlichkeit der Beibehaltung der falschen Anfangsüberzeugung von $56 \%$ auf $40 \%$, wenn die Probanden vor dem Korrekturversuch in ihrem Selbstwertgefühl gestärkt wurden. 
„For example, it no longer matters whether people are misinformed about climate science if they adopt ecologically friendly behaviors, such as by driving low-emission vehicles, in response to "nudges", such as tax credits." (Lewandowsky et al 2012, 124; kursiv von PK)

An dieser Stelle zeigt sich das verschärfte Paternalismusdilemma: Der Versuch, die Erkenntnisse über „Backfire Effects“ konstruktiv für die Praxis der Irrtumskorrektur einzusetzen, resultiert in der Empfehlung einer unterkomplexen Vermittlungspraxis, Überredungsmechanismen und tendenzieller Unterschlagung relevanter Argumente. Die Konsequenz wäre, die Akteure nicht mehr als Adressaten für Aufklärungsbemühungen ernst zu nehmen, sondern sie durch Manipulation zum richtigen Handeln zu bewegen.

Eine solche Manipulation der Akteure mag zwar aufklärungstechnologische Erfolgschancen erhöhen, steht jedoch in praktischem Widerspruch zum zusätzlichen Ziel der Förderung der Autonomie und Mündigkeit der Akteure - falls man dieses Ziel denn verfolgen will. Ist jedoch die Förderung von Autonomie und Mündigkeit eines der Ziele ideologiekritischer Praxis, so verschärft sich das logische Paternalismusdilemma in der Vermittlung kritischer Kompetenzen nun durch die unerwarteten Konsequenzen der Verbesserung der Erfolgsbedingungen aufklärungstechnologischer Bemühungen und ihrer Beurteilung mittels der Berücksichtigung relevanter Ergebnisse der empirisch-experimentell verfahrenden Einzelwissenschaften. Wie anfangs erwähnt ist der Rückgriff auf diese Wissenschaften einerseits unerlässlich für die Erfolgsaussichten ideologiekritischer Praxis, andererseits resultieren daraus mitunter unerwartete und wenig beachtete Probleme - wie das Dilemma zwischen aufklärerischer Erfolgswahrscheinlichkeit und dem Ziel der ideologiekritischen Förderung von Mündigkeit und Autonomie.

Dieser praktische Widerspruch, der sich aus jenem Zielkonflikt zwischen dem Wunsch nach erfolgreichen aufklärungstechnologischen Methoden einerseits und dem Zweck der Förderung der Autonomie und Mündigkeit der Akteure andererseits ergibt, kann allerdings im Folgenden aufgelöst werden. Zuvor möchte ich die bisher skizzierten aufklärungstechnologischen Perspektiven zusammenfassen. 


\section{Fazit I: Aufklärungstechnologische Perspektiven}

Es wurde gezeigt, dass die Methode der Bildungsvermittlung in jeder Aufklärungstechnologie zumindest berücksichtigt werden muss und welche unerlässliche Rolle die empirischen und idealiter experimentell verfahrenden Wissenschaften hier - einerseits als Inhalt entsprechender Bildung, andererseits als Instrument der Einschätzung der Erfolgsbedingungen aller aufklärungstechnologischer Bemühungen - spielen müssen. Die Idee der Selbstbefreiung durch das Wissen als ideelle Aufklärungstechnologie kann anhand der folgenden drei Eigenschaften charakterisiert werden:

Erstens durch wissenschaftliche Praxis zu relevanten Forschungsgegenständen, was eine gewisse institutionalisierte Forschungsfreiheit samt sicherer Finanzierung voraussetzt. Die emanzipationsrelevanten Forschungsgegenstände sind hierbei vordringlich solche aus den akademischen Disziplinen der Psychologie und Soziologie, da sie menschliches Denken etwa in Abhängigkeit von seinen Rahmenbedingungen und seiner Fehleranfälligkeit zum Gegenstand haben.

Zweitens durch adäquate Wissenschaftsvermittlung über erkenntniskritische und ideologiekritische Themen, insbesondere solche der "unvollständigen Rationalität" , Denkfehler und sozio-epistemischen Abhängigkeiten, sowie einem erkenntnis- und wissenschaftstheoretischen Methodenrüstzeug, verbunden mit der Lehre der Distanzierung von den eigenen Ideen, um sie kritisch zum Gegenstand machen zu können. Zugleich sind möglichst geringe Hürden für den Zugang zu entsprechender emanzipationsrelevanter Bildung nötig, denn kritisches Denken setzt spezifisches Sachwissen über kritisches Denken voraus.

Drittens durch die Forderung nach angemessenen institutionellen Rahmenbedingungen und Lebensumständen politischer, ökonomischer und sozialer Art, die es überhaupt erst erlauben, dass es für die aufzuklärenden Akteure binnenrational ist, sich dem Erlernen kritischer Kompetenzen und der praktischen Korrektur ihrer Überzeugungen zu widmen. Mitunter mag eine Bereitschaft zur Irrtumskorrektur zudem davon abhängen, ob die Lebensumstände der Akteure die Aufrechterhaltung eines positiven Selbstwertgefühls erschweren (Fußnote 22). Andernfalls wäre die Konsequenz, dass die Aufklärungsbemühungen versanden, da die „erlernten" Kenntnisse und Methoden nicht in die Praxis der Akteure überführt würden. 
Hier zeigt sich, dass die starre Dichotomie zwischen ideeller und materieller Aufklärung ein Scheinproblem darstellt: So wie materielle Aufklärung unter der Gefahr der Selbstwidersprüchlichkeit und Fruchtlosigkeit nicht ohne die Berücksichtigung ideeller Aufklärung konzipiert sein kann, kann ideelle Aufklärung ohne materielle Aufklärung das von ihr bereitgestellte und vermittelte Wissen nicht erfolgreich in die Lebenspraxis der aufzuklärenden Akteure überführen. Der anfangs kritisierte Methodenmonismus ist also in jedem Falle ein Problem, das dementsprechend auch Programme ideeller Aufklärung trifft und die Öffnung des Spielraums erfolgsversprechender ideologiekritischer Strategien erzwingt. Dementsprechend könnte diese Erkenntnis eine Gelegenheit darstellen, die ideologiekritischen Programme der ideellen, an der klassischen Aufklärungsphilosophie und ihrer Bildungsvermittlung orientierten Aufklärungstechnologie zumindest teilweise mit den die menschlichen Lebensbedingungen betonenden marxistischen Konzepten zu versöhnen, da sie in Teilen aufeinander angewiesen sind.

Man darf jedoch nicht dem Trugschluss aufsitzen, mit einer solchen Perspektive bereits alle Instrumente einer erfolgreichen Aufklärungstechnologie an der Hand zu haben. Einerseits müssen empirische Erkenntnisse berücksichtigt werden, die ein so skizziertes aufklärungstechnologisches Verfahren problematisieren und ggf. auf bestimmte Anwendungsfälle einschränken können. Andererseits können sich durch einen solchen Rückgriff auf empirische Erkenntnisse Zielkonflikte ergeben: Denn vor dem Hintergrund von „Backfire Effects“ etwa stellt sich nun die Frage, in welchem Maße wir Manipulation, Überredungsstrategien und rhetorische "Tricks" in der Wissensvermittlung einsetzen sollten, wenn wir uns am Ideal der zu befördernden Autonomie der Akteure orientieren wollen.

\section{Fazit II: Vermeidung des Paternalismusdilemmas}

Zuletzt sind deshalb das en passant aufgeworfene Paternalismusdilemma in seiner durch die Berücksichtigung der „Backfire Effects“ verschärften Form und die Perspektiven zu seiner Vermeidung aufzugreifen.

Das paternalistische Moment der ideellen Aufklärungstechnologie als "Selbstbefreiung durch das Wissen" ist analog zu sehen zu der entwicklungspsychologisch notwendigen Voraussetzung eines kindlichen Urvertrauens, um im späteren Leben einen rationalen Umgang mit Vertrauens- 
beziehungen an den Tag legen zu können. Die Emanzipation scheint in beiden Fällen - Aufklärung hier und Erwerb von Vertrauenskompetenzen dort - eine extern angestoßene "Hilfe zur Selbsthilfe“ zu sein. Das Ziel ist in beiden Fällen die Entwicklung der Autonomie des Individuums. Auch beim frühkindlichen Erwerb von Vertrauenskompetenzen ist ein unkritisches, „primitives“ Vertrauen die notwendige Voraussetzung für den Erwerb derjenigen Kompetenzen, die eine kritische Prüfung jenes ursprünglichen Vertrauensverhältnisses erst ermöglichen:

„Die Fähigkeiten, die wir brauchen, um die Äußerungen anderer auf ihre Wahrheit hin zu überprüfen, werden in Lehrsituationen erworben, in denen diese Fähigkeiten noch nicht vorhanden sind. [...] [Unkritisches] Vertrauen dient in diesem Sinne als Grundlage für den Erwerb kritischer Beurteilungskompetenz, ist aber primitiv, weil es selbst noch voraussetzungslos gegeben ist und keiner kritischen Überprüfung bedarf." (Hartmann 2011, 66f.)

Der Erwerb kritischer Kompetenzen ist also tatsächlich paternalistisch: Die Autonomie lässt sich nur durch eine Anfangsphase der Unmündigkeit erwerben; anschließend liegen die kritischen Instrumente vor, um entsprechende Verhältnisse angemessen beurteilen zu können - inklusive des anfänglichen Verhältnisses der paternalistischen Vermittlung.

Für die Zielkonflikte bei der Vermeidung von „Backfire Effects“ bedeutet dies, dass es tatsächlich unproblematisch sein dürfte, auf bestimmte Manipulationen zurückzugreifen, insofern sie einzig und allein dazu genutzt werden, um kritische, methodische Kompetenzen und ideologiekritisches Sachwissen zu vermitteln, die es dem Akteur nachträglich erlauben, das Manipulationsverhältnis und ähnliche Kontexte zu durchschauen und zu problematisieren. Das hierzu nötige Wissen besteht insbesondere in ideologiekritischem Wissen selbst: Das Wissen, das die Ideologiekritikerinnen und Aufklärerinnern für ihr eigenes Unterfangen und dessen Optimierung verwenden, ist es, dessen Vermittlung der Förderung kritischer Urteilskompetenzen und damit der Autonomie der aufzuklärenden Akteure in besonderem Maße zuträglich ist.

Das Sachwissen über die unvollständige Rationalität, vor allem aus den wissenschaftlichen Disziplinen der Psychologie, Soziologie sowie Erkenntnis- und Wissenschaftstheorie erfüllt also drei aufklärungstechnologische Funktionen: Erstens ist es im Rahmen ideologiekritischer Theorien nötig, um Ideologien - ihre Entstehung, Persistenz und Konsequenzen zu erklären. Zweitens ist es nötig, um aufklärungstechnologische Erfolgs- 
bedingungen beurteilen und optimieren $\mathrm{zu}$ können, worunter auch Aspekte materieller Aufklärung fallen, um die ideelle Aufklärungsprogramme unter Berücksichtigung der ihnen eigenen Grenzen erfolgreicher ideologiekritischer Praxis zu ergänzen sind. Und drittens stellen sie den nötigen Inhalt der Vermittlung und Förderung kritischer Kompetenzen: Wem an der Förderung von Autonomie, Mündigkeit und damit kritischer Urteilskompetenzen und Desillusionierung gelegen ist, muss auf empirisch und idealiter experimentell qualifiziertes Sachwissen über die unvollständige Rationalität und die Faktoren kognitiver Fehlleistungen zurückgreifen - auch um die Notwendigkeit bestimmter materieller Aufklärungs- und möglicher Manipulationsstrategien zu ermitteln - und allein schon zur Vermeidung des Paternalismusdilemmas muss eben dieses ideologiekritische Sachwissen der Inhalt jeder ideellen Aufklärungstechnologie sein.

\section{Literatur}

Albert, Hans. 1960. „Wissenschaft und Politik. Zum Problem der Anwendbarkeit einer wertfreien Sozialwissenschaft". In Probleme der Wissenschaftstheorie, Festschrift für Victor Kraft, herausgegeben von Ernst Topitsch, 201-232. Berlin/Heidelberg: Springer.

Baumann, Peter. 2006. Erkenntnistheorie. Stuttgart: Metzler.

Bohlender, Matthias. 2010. „Die Herrschaft der Gedanken. Über Funktionsweise, Effekt und die Produktionsbedingungen von Ideologien". In Karl Marx und Friedrich Engels: Die deutsche Ideologie. Klassiker auslegen (Band 36), herausgegben von Harald Bluhm, 42-57. Berlin: Akademie-Verlag.

Boudon, Raymond. 1988. Ideologie. Geschichte und Kritik eines Begriffs. Reinbek bei Hamburg: Rowohlt.

Cook, John und Stephan Lewandowsky. 2012 [2011]. The Debunking Handbook. St. Lucia, Australia: University of Queensland.

Eagleton, Terry. 2000 [1993]. Ideologie. Eine Einführung. Stuttgart/Weimar: J.B. Metzler.

Elster, Jon. 1986. An Introduction to Karl Marx. Cambridge: Cambridge University Press.

Elster, Jon. 1987. Subversion der Rationalität. Frankfurt/New York: Campus.

Gosepath, Stefan. 1992. Aufgeklärtes Eigeninteresse. Frankfurt am Main: Suhrkamp.

Gröbl-Steinbach Schuster, Evelyn. 2011. „Was will und was kann Ideologiekritik?“ In Philosophie und Wirtschaftswissenschaften, herausgegeben von Volker Gadenne und Reinhard Neck, 179-197. Tübingen: Mohr Siebeck.

Hartmann, Martin. 2011. Die Praxis des Vertrauens. Berlin: Suhrkamp. 
Keutner, Thomas. 2004. Ignoranz, Täuschung, Selbsttäuschung. Kausalität in den Handlungswissenschaften. Freiburg/München: Karl Alber.

Körner, Patrick. 2015. „Erklärungsprobleme erkenntniskritischer Ideologietheorien“. Mythos-Magazin. http:// www.mythos-magazin.de/ideologieforschung/pk_erkl.pdf. Zugegriffen: 01.07.2019.

Körner, Patrick. 2017. „Zur Beurteilung von Verschwörungstheorien“. Mythos-Magazin. http:// www.mythos-magazin.de/ideologieforschung/pk_verschwoerungstheorien.pdf. Zugegriffen: 01.07.2019.

Körner, Patrick. 2018. „Irrwege marxistischer Ideologiekritik“. Mythos-Magazin. http://www.mythos-magazin.de/ideologieforschung/pk_marx.pdf. Zugegriffen: 01.07.2019

Lewandowsky, Stephen, Ulrich Ecker, Colleen Seifert, Norbert Schwarz, und John Cook. 2012. "Misinformation and Its Correction: Continued Influence and Successful Debiasing." Psychological Science in the Public Interest 13(3), 106-131.

Marx, Karl und Friedrich Engels. 1958/1978 [1845-1846]. „Die deutsche Ideologie“. In Werke $(M E W)$, Band 3, 5-530. Berlin/DDR: Dietz.

Nyhan, Brendon und Jason Reifler. 2010. „When corrections fail: The persistence of political misperceptions." Political Behavior 32, 303-330.

Nyhan, Brendon und Jason Reifler. 2017. „Blank slates or closed minds? The role of information deficits and identity threat in the prevalence of misperceptions." http:// www.dartmouth.edu/ nyhan/opening-political-mind.pdf. Zugegriffen: 01.07.2019.

Popper, Karl. 2006 [1987]. Auf der Suche nach einer besseren Welt. 14. Auflage. München: Piper.

Salamun, Kurt. 1991. „Der Ethos der Aufklärung im kritischen Rationalismus“. In Moral und Politik aus der Sicht des kritischen Rationalismus, herausgegeben von Kurt Salamun, 95119. Amsterdam: Rodopi.

Schmid, Michael. 2004. „Ideologie und Rationalität. Bemerkungen zu einem Erklärungsproblem der Weltanschauungsanalyse." Aufklärung und Kritik 8, 215-232.

Taber, Charles S. und Milton Lodge. 2006. „Motivated Skepticism in the Evaluation of Political Beliefs". American Journal of Political Science 50(3), 755-769.

Tepe, Peter. 2012. Ideologie. Berlin/Boston: de Gruyter.

\section{Open Access}

Dieser Beitrag erscheint unter der Creative-Commons-Lizenz CC-BY-SA 4.0:

https://creativecommons.org/licenses/by-sa/4.0/ 